\title{
Interinstitutional agreement of individual functional independence measure (FIM) items measured at two sites on one sample of SCI patients
}

\author{
M E Segal PhD, * J F Ditunno MD, W E Staas MD \\ Regional Spinal Cord Injury Center of Delaware Valley at Thomas Jefferson University, \\ 132 South 10th St, 375 Main, Philadelphia, PA 19107, USA.
}

Individual items of the Functional Independence Measure (FIM) were analyzed for interinstitutional agreement using a sample of 57 spinal cord injured (SCI) patients at discharge from an acute care rehabilitation setting (ACRS) and at admission to an ongoing rehabilitation setting (ORS). The two FIM ratings were performed within 6 days of each other.

The reliability coefficient for total FIM scores was good: 0.83. Individual items were classified into four groups: (1) above average reliability coefficient, above average proportion agreement, which included the feeding items and items from the mobility category; (2) above average reliability coefficient, below average agreement, which included the majority of items in the selfcare category; (3) below average reliability coefficient, above average agreement, which included items in the sphincter control and communication categories; and (4) below average reliability coefficient, below average agreement, which included items in the social cognition category. Patients received significantly higher ratings on most selfcare items in the ACRS, and significantly higher ratings on social cognition items in the ORS. Implications of these results are discussed in terms of using individual FIM items for research purposes.

Reliability coefficients were acceptably high for subgroups of complete and incomplete paraplegics and for complete quadriplegics, ranging from 0.74 to 0.87 , but low for incomplete quadriplegics, $r=0.49$. However, these sample sizes were small, ranging from 9 to 17 patients. Data collection will continue in order to increase sample size and permit further analysis of these subgroups.

Keywords: spinal cord injury; FIM reliability; FIM interinstitutional agreement.

\section{Introduction}

The Functional Independence Measure (FIM) was implemented, after extensive testing for reliability and validity by the Uniform Data System (UDS), headquartered in Buffalo, NY, in the mid-1980s to measure functional status of rehabilitation patients. It was developed for rehabilitation patients with varying disabilities, and includes 18 items grouped into six categories: selfcare, sphincter control, mobility, locomotion, communication, and social cognition. Measurement of each of the 18 items is on a 7 -point scale, with 1 corresponding to

*Correspondence: Moss Rehabilitation Research Institute, Moss Rehabilitation Hospital, 1200 West Tabor Road, Philadelphia, PA 19141, USA. complete dependence and 7 to complete independence (see Table I). The FIM is now used at over 100 institutions in the United States and in several countries abroad. The intent of the FIM developers was to create an instrument to assess 'burden of care', defined as the 'substituted time/energy which must be brought to serve the dependent needs of the disabled individual so that a certain quality of life may be achieved and maintained' during and after rehabilitation; to this end, a patient's scores on the 18 items are summed to produce a total score. ${ }^{1}$ In other words, as originally conceived, development of the FIM was driven by patient care needs.

Recently, there has been increased interest in using the FIM as a research tool. The 13 Federally-funded regional spinal 
Table I General description of FIM items' levels of function and their scores

\begin{tabular}{|c|c|c|}
\hline Score & Level of function & General description \\
\hline 7 & Complete independence & $\begin{array}{l}\text { All of the tasks described as making up the activity are } \\
\text { typically performed safely, without modification, assistive } \\
\text { devices, or aids, and within reasonable time }\end{array}$ \\
\hline 6 & Modified independence & $\begin{array}{l}\text { Activity requires any one or more than one of the follow- } \\
\text { ing: an assistive device, more than reasonable time, or } \\
\text { there are safety (risk) considerations }\end{array}$ \\
\hline 5 & Supervision or setup & $\begin{array}{l}\text { Subject requires no more help than standby, cuing or } \\
\text { coaxing, without physical contact. Or, helper sets up } \\
\text { needed items or applies orthoses }\end{array}$ \\
\hline 4 & $\begin{array}{l}\text { Minimal contact assist- } \\
\text { ance }\end{array}$ & $\begin{array}{l}\text { With physical contact, the subject requires no more help } \\
\text { than touching, and subject expends } 75 \% \text { or more of the } \\
\text { effort }\end{array}$ \\
\hline 3 & Moderate assistance & $\begin{array}{l}\text { Subject requires more help than touching, or expends half } \\
(50 \%) \text { or more (up to } 75 \% \text { ) of the effort }\end{array}$ \\
\hline 2 & Maximal assistance & $\begin{array}{l}\text { Subject expends less than } 50 \% \text { of the effort, but at least } \\
25 \%\end{array}$ \\
\hline 1 & Total assistance & Subject expends less than $25 \%$ of the effort \\
\hline
\end{tabular}

cord injury (SCI) model systems collect FIM data for their national database after having conducted an interrater reliability trial among participating centers. ${ }^{2}$ The National Acute Spinal Cord Injury Study (NASCIS) has incorporated the FIM into the NASCIS III study design as an outcome measure in testing the effectiveness of drug interventions for acutely injured SCI patients. ${ }^{3}$ There has been some success in using individual FIM items in monitoring quality issues in hospital, eg patient scores on the FIM bed transfer item have been related to number of falls on a rehabilitation unit over time. ${ }^{4}$ The dimensions represented by the FIM items have been explored by investigators using models derived from item response theory, ${ }^{5}$ a well documented approach in the testing and measurement literature which takes into account the probability of a test-taker earning a particular score on a particular item on a test. ${ }^{6}$ Perhaps most importantly, FIM scores will now be included in the 1992 revised ASIA standards for neurological and functional classification of SCI, ${ }^{7.8}$ which promise an agreed-upon international taxonomy for classifying severity of SCI. ${ }^{9}$

In this light, questions need to be answered not only about interrater reliability of the FIM, which has been investigated with good results by Hamilton et al, ${ }^{10}$ but also about the underlying structure of the FIM items when considered in an interinstitutional context. In other words, we need to know not only whether raters at one site use the FIM scale reliably and appropriately, but also whether appropriate use of the scale results in the same FIM item rating at two sites for the same patient when measured within a short enough interval so that his/her real functional abilities have not changed.

In this paper, we suggest a methodology to begin to understand this structure, as well as to answer some questions about the appropriateness of various FIM items for specific patient populations, in this case SCI. We use data gathered retrospectively at the Regional Spinal Cord Injury Center of Delaware Valley (RSCICDV) in Philadelphia. The RSCICDV consists of two institutions: the 32-bed rehabilitation unit of a large (717-bed) inner city university hospital where patients are admitted acutely, and a 97-bed free-standing rehabilitation hospital nearby where patients transfer for ongoing rehabilitation. Both sites collect FIM data within 3 days of both admission and discharge of patients to the SCI rehabilitation units for submission to UDS. At both sites, the items are discipline-specific; eg 
physical therapists collect data on the mobility and locomotion items, occupational therapists on the selfcare items, and nursing on the sphincter control items. Admission FIM data from the acute care rehabilitation setting (ACRS) and discharge data from the ongoing rehabilitation setting (ORS) are sent to the SCI model systems' national database in Birmingham, Alabama.

Recently, we recognized that discharge data from the ACRS and admission data to the ORS, which are collected within a maximum of 6 days of each other on SCI patients, were available and could provide a naturally-occurring field study of interinstitutional agreement of individual FIM items as well as of total FIM scores.

\section{Issues raised by reliability theory}

In such a study, at least five sources of potential variation exist for individual FIM items measured at two different sites on the same sample of patients within 6 days:

1 Poor item construction produces unsystematic rating error.

2 Raters at one institution use the scale in a systematically different way from raters at the other institution, either through misunderstanding of scale items or through 'leniency' or 'severity' effects.

3 The patient's motivational state changes across sites.

4 The actual physical strength or skill of the patient changes during the time interval between the two evaluations. In this case, it is unlikely that a 6 day interval would produce major changes in individual FIM item ratings, but not impossible.

5 Environmental differences and differences in patient management protocols between the two sites lead to differences in individual FIM item ratings.

In classical reliability theory, (1) and (2) above correspond to issues raised by interrater reliability; (3) and (4) are factors that should be minimized in test-retest reliability; and (5) represents a kind of 'alternateform' reliability. ${ }^{11}$ All three forms of variation are potentially present for the FIM items in this study.

We point out at the outset that the data were collected without benefit of a systematic training program at either site; neither site had been credentialled according to standard UDS procedures. This situation has since been corrected with in-services available monthly; all FIM data collectors are required to pass an in-house mastery examination, and the sites are now credentialled by UDS. We believe it is reasonable to assume that the patterns in these data may be representative of data from institutions which may not routinely emphasize FIM mastery and reliability. However, no data were available at the time of this study on interrater agreement within each of the sites.

The major question the study sought to address was: How can we begin to disentangle the effects listed above, given one score from each patient on each item from each of the two sites within a maximum of 6 days of each other? A second question is: How can we determine which FIM items are most appropriate for research questions involving a specific patient population (here, SCI)?

One way to sort out the various sources of score variation would be to consider them one at a time, as pointed out by Anastasi in her classic text on testing. ${ }^{11}$ Using this scenario, patients would be evaluated four times: (1) by different raters at the same time and site to determine interrater reliability within a site; (2) by the same raters, evaluating the same patients at both sites within a day or two of transfer to determine interinstitutional environmental differences and site-dependent differences in patient motivation; (3) by the same rater at the same site after an interval of 6 days to determine the appropriateness of a 6-day test-retest interval; and (4) by raters from both sites evaluating the same patient at the same place and time to determine if systematic differences exist in raters' motivation and use of the scale between sites. These sources of variation could then be compared and components of variances subtracted from total variance.

However, in healthcare settings, given the demands of patient care, such procedures are often unaffordable luxuries, and we must compromise in favor of useful but less 
rigorous analyses. In this situation, a way to begin to do this is to compare results produced by two different methods of measuring agreement: the reliability coefficient and a statistic measuring the proportion of exact agreement.

The reliability coefficient was first suggested as a measure of agreement by Spearman in his classic treatment of reliability. ${ }^{12}$ It is nothing more than a Pearson productmoment correlation coefficient computed on the two test administrations; however, its interpretation is quite different. While the Pearson correlation coefficient is squared to produce a proportion representing the amount of variability in one variable which can be 'accounted for' by the variability in the other (and vice versa), the reliability coefficient has a direct interpretation, without squaring its value, as the ratio of the variance of the 'true scores' of the test takers to the variance of the observed scores. 'True scores' are assumed to be the scores the test takers would receive if the testing instrument and situation were free of measurement error; this error is assumed to exist to a greater or lesser extent on every test, and can be quantified by the various psychometric properties of the test. Interested readers are referred to well known descriptions of reliability theory ${ }^{6.11}$ for further explanation. For purposes here, a reliability coefficient of 0.60 indicates that $60 \%$ of the amount of score differences observed among test takers in a single test administration can be attributed to 'true score' variance, and that $40 \%$ of the variance in the scores represents measurement error.

There is a problem with relying exclusively on a reliability coefficient to assess rater agreement: because it has the properties of a correlation coefficient, it represents agreement on relative standing of subjects within the group of subjects from one test to another, and not absolute one-to-one correspondence of ratings. Therefore, systematic differences in scoring from one test to another will not be picked up, and will result in high reliability coefficients. Using a FIM item as an example, if every patient who was rated 1 at the first site received 3 at the second, every patient rated 2 at the first site received 4 at the second, and so on, the correlation would be perfect, because relative standing in the group was consistent across the two sites.

A different approach is possible using the kappa statistic, first formulated by Cohen. ${ }^{13}$ Unlike the reliability coefficient, kappa offers a direct measurement of rater agreement, corrected for chance. However, kappa is inappropriate for some FIM item data collected on SCI patients because the marginals (column and row totals) in the matrix used to calculate kappa are unbalanced $;^{14}$ ie a high proportion of SCI patients score either very high or very low on many FIM items. This finding was reported by Davidoff $e t a l^{15}$ for items on the communication and social cognition subscales. Kappa underrepresents the proportion of agreement when the marginal totals are unbalanced because the correction for chance agreement is calculated by taking the marginal totals into account in the formula. ${ }^{14}$

It appears we are left with a less than ideal strategy for measuring rater agreement: the simple proportion of scores that are in agreement on a particular item. The problem with this method is that it fails to take into account chance agreement between ratings; however, it appears to be the best available method for these data if we wish to compare actual score agreement with the Pearson correlation, or reliability coefficient.

\section{Methods}

Fifty-seven subjects were identified who had (1) received treatment at the RSCICDV between January 1989 and May 1990, (2) transferred without interruption of care directly from the ACRS to the ORS, and (3) were without missing FIM discharge data from the ACRS or missing FIM admission data to the ORS. FIM data collections at the two sites fell within a maximum of 6 days of each other.

Means and standard deviations for each of the FIM items at each of the two sites were calculated. 
Two measures were calculated on total scores and on scores for individual FIM items: Pearson product-moment correlation coefficients, to yield reliability coefficients, and the proportion of agreement, as described earlier.

Each FIM item was then assigned to one category of a four-way classification: (1) above average reliability coefficient, above average proportion agreement; (2) above average reliability coefficient, below average proportion agreement; (3) below average reliability coefficient, above average proportion agreement; and (4) below average reliability coefficient, below average proportion agreement.

FIM items were also analyzed with dependent $t$-tests to determine whether scores upon transfer to the ORS were significantly higher or lower than at the ACRS.

Finally, reliability coefficients for FIM total scores for four separate groups of patients (quadriplegic complete, quadriplegic incomplete, paraplegic complete, and paraplegic incomplete) were calculated. Because of the small sample sizes of these subgroups, further analyses were not undertaken.

\section{Results}

The mean of the total FIM scores in the ACRS was 65.31. Their mean in the ORS was 66.22 , a nonsignificant difference. Table II presents the means and standard deviations for the 18 individual FIM items measured at each of the two sites.

Reliability for total FIM scores across the two settings was 0.83 . Table III presents the reliability coefficients and the proportion of agreement for each of the 18 individual FIM items. The median reliability coefficient for these individual items was 0.42 . The median proportion agreement for the items was 0.465 .

On the basis of Table III, the FIM items were classified into four groups, above and below the median reliability coefficient and median proportion agreement as described earlier. Results of this classification are presented in Table IV.

Dependent $t$-tests on the FIM items produced significant differences for 10 items: most of the selfcare items (except for toileting) and ability to maneuver walking or in a wheelchair were rated consistently higher at the ACRS, and toileting and the

Table II Means and standard deviations of FIM items in acute and ongoing rehabilitation settings

\begin{tabular}{lcccc}
\hline Item & \multicolumn{2}{c}{ Acute setting } & \multicolumn{2}{c}{ Ongoing rehabilitation } \\
\cline { 2 - 3 } & Mean & SD & Mean & SD \\
\hline Feeding & 5.30 & 1.90 & 4.49 & 2.46 \\
Grooming & 4.95 & 2.07 & 4.30 & 2.50 \\
Bathing & 3.26 & 1.96 & 2.58 & 1.83 \\
Upper dressing & 3.88 & 2.10 & 3.26 & 2.29 \\
Lower dressing & 2.53 & 1.65 & 2.07 & 1.40 \\
Toilet & 1.56 & 1.30 & 1.95 & 1.25 \\
Bladder & 1.44 & 1.28 & 1.77 & 1.43 \\
Bowel & 1.51 & 1.38 & 1.72 & 1.35 \\
Bed transfer & 2.65 & 1.85 & 2.40 & 1.50 \\
Toilet transfer & 2.19 & 1.54 & 2.33 & 1.49 \\
Tub transfer & 1.86 & 1.43 & 2.14 & 2.35 \\
Walk/wheelchair & 3.88 & 2.31 & 3.18 & 1.17 \\
Stairs & 1.07 & 0.53 & 1.26 & 0.48 \\
Comprehension & 6.84 & 0.62 & 6.86 & 0.53 \\
Expression & 6.82 & 0.63 & 6.84 & 1.42 \\
Social interaction & 5.00 & 1.57 & 6.21 & 1.17 \\
Problem solving & 5.04 & 1.60 & 6.23 & \\
Memory & 5.53 & 1.43 & 6.63 & \\
\hline
\end{tabular}


Table III Reliability coefficient and proportion agreement for individual FIM items

\begin{tabular}{lcc}
\hline Item & $\begin{array}{c}\text { Reliability } \\
\text { coefficient }\end{array}$ & $\begin{array}{c}\text { Proportion } \\
\text { agreement }\end{array}$ \\
\hline Feeding & 0.77 & 0.61 \\
Grooming & 0.63 & 0.42 \\
Bathing & 0.62 & 0.32 \\
Upper dressing & 0.67 & 0.32 \\
Lower dressing & 0.46 & 0.42 \\
Toilet & 0.35 & 0.46 \\
Bladder & 0.10 & 0.65 \\
Bowel & 0.16 & 0.63 \\
Bed transfer & 0.72 & 0.47 \\
Toilet transfer & 0.65 & 0.60 \\
Tub transfer & 0.60 & 0.63 \\
Walk/wheelchair & 0.62 & 0.44 \\
Stairs & 0.32 & 0.95 \\
Comprehension & 0.08 & 0.81 \\
Expression & 0.02 & 0.81 \\
Social interaction & 0.25 & 0.23 \\
Problem solving & 0.30 & 0.19 \\
Memory & 0.39 & 0.32 \\
\hline
\end{tabular}

three social cognition items were rated significantly higher at the ORS. These results are presented in Table $\mathrm{V}$.

All of these 10 items were not rated consistently in one direction at either setting; in fact, the ratings were split $60 / 40 \%$, with six of the individual item ratings higher at the ACRS and four of them higher at the ORS. However, the items rated consistently higher at the ACRS consisted of five of the six selfcare items and a locomotion item, and the items rated consistently higher at the ORS were all of the items in the social cognition category and one from the selfcare category.

Finally, the reliability coefficient calculated for the sample of 14 complete quadriplegic patients was 0.87 ; for the sample of 17 incomplete quadriplegic patients, 0.49 ; for the sample of 13 complete paraplegic patients, 0.74; and for the sample of nine incomplete paraplegic patients, 0.85 . Three of the patients included in the total sample were excluded from these analyses because they had multiple injuries including head injuries, and the level of injury severity for one of the patients had not been coded.

Further analyses of these subgroups were not undertaken because of small sample sizes.

\section{Discussion}

The median reliability score for the 18 individual FIM items was considerably lower than the reliability for the total FIM scores: 0.42 vs 0.83 . This is not a particularly surprising result, as reliability theory tells us that all other things being equal, the longer a test, the higher the reliability (in this case, the total FIM score represents a test 18 times as long as any one individual FIM item). A reliability coefficient of 0.83 is well within the bounds of good reliability; thus, total scores reflecting burden of care appear stable and consistent between the two sites.

The picture is less clear-cut for the individual items. We have grouped them into the four categories described above.

\section{Group 1: Above average reliability coefficient; above average proportion agreement}

These four items (feeding, bed transfers, toilet transfers, and tub transfers) were stable across time and sites. Raters achieved an acceptable number of ratings with exact agreement. In FIM training and mastery sessions at the RSCICDV, the feeding and bed transfer items have been the easiest for subjects to score. ${ }^{16}$

\section{Group 2: Above average reliability coefficient; below average proportion agreement}

These six items (grooming, bathing, upper body dressing, lower body dressing, toileting, and walk/wheelchair) showed systematic variation in the way the scale was used across the two sites. Although exact ratings did not agree, patients rated high at one institution tended to be rated high at the other, and patients rated low at one institution tended to be rated low at the other. Reasons for the systematic disagreement could be due to changes in patient motivation, patient management, or tendencies of staff at one institution to rate patients higher (or lower) than at the other institution.

Discussions with FIM coordinators at both institutions could not uncover differences which might be due to patient management. If anything, the environment at 
Table IV Comparison of FIM items' interinstitutional agreement by relating reliability coefficients to proportion of agreement

Above average reliability coefficients, above average agreement

Feeding

Bed transfers

Toilet transfers

Tub transfers

The scale was used consistently across the two institutions, and any differences in administering the items and collecting the data were small

Above average reliability coefficients, below average agreement

Grooming

Bathing

Upper dressing

Lower dressing

Toileting

Walk/wheelchair

Exact scores on these items differed, although the differences appear to be a result of consistent, systematic differences in the way the scale was administered at the two points in time at the two institutions

Below average reliability coefficients, above average agreement

Bladder management

Bowel management

Stairs

Comprehension

Expression

Agreement was acceptable across the two sites, although a lack of variability in the scores of SCI patients contributed to low reliability coefficients

Below average reliability coefficients, below average agreement

Social interaction

Problem solving

Memory

Differences in exact scores on these items were not related to systematic differences in the way the scale was administered, and are probably due to confusion among raters about meaning of scale values

Table V FIM items which differed significantly across sites*

\begin{tabular}{lccc}
\hline Item & $t$-test & $p$ & Site with higher score \\
\hline Feeding & 3.87 & 0.0003 & Acute \\
Grooming & 2.46 & 0.0172 & Acute \\
Bathing & 3.11 & 0.0029 & Acute \\
Upper dressing & 2.60 & 0.0118 & Acute \\
Lower dressing & 2.16 & 0.0349 & Acute \\
Toilet & 2.01 & 0.0491 & Ongoing rehab \\
Walk/wheelchair & 2.59 & 0.0121 & Acute \\
Social interaction & 4.97 & 0.0001 & Ongoing rehab \\
Problem solving & 4.83 & 0.0001 & Ongoing rehab \\
Memory & 5.76 & 0.0001 & Ongoing rehab \\
\hline
\end{tabular}

*All results were tested for two-tailed levels of significance, $d f=56$. 
the ORS would tend to promote higher scores in selfcare activities, whereas scores at the ORS actually produced lower scores for all of the selfcare items except toileting.

An example of how the environment at the ORS might have produced higher scores may be illuminating here. At the ACRS, all SCI quadriplegic patients perform grooming activities at the bedside, requiring an aide to provide them with water, washcloth, etc. Thus, their maximum score on this item would be 5 , requiring setup (see Table I). At the ORS, patients' rooms have sinks equipped with electronic beams which turn the water on when the patient breaks the beam by passing his/her hand across it. Thus, a patient who required setup in the ACRS might be independent in the ORS.

As described above, a pattern opposite to the one suggested in this example actually occurred in the data. Two possibilities remain: patient motivation changed, or raters were using the scale in systematically different ways.

In our analysis, either of these appear possible. Patients are in a new and thus unfamiliar environment at the time of FIM testing in the ORS, and thus may be more cautious and fearful. On the other hand, there may be a tendency for staff at admission to rate with more severity, and for staff at discharge to rate with more leniency, and the data may be tapping into this propensity. These two competing explanations are confounded in these data, and further discussion and testing are necessary to disentangle them.

\section{Group 3: Below average reliability coefficient, above average proportion agreement}

These five items (bladder management, bowel management, stairs, comprehension, and expression) showed good agreement in their scoring across sites. The reason for the poor reliability coefficients is a restriction of range of scores. In other words, almost all patients performed either very well or very poorly on these items, a situation which restricts the correlation coefficient range and renders it very unstable. For example, the items on bladder management, bowel management, and stairs were low for almost all patients; items on comprehension and expression were high for almost all patients (see Table II for means and standard deviations of these items).

\section{Group 4: Below average reliability coefficient, below average proportion agreement}

These three items, all on the social cognition subscale (social interaction, problem solving, and memory) received different ratings by the FIM scorers at the two sites, and there was no discernible pattern in the ratings. These data lead to the suggestion that these items are the most difficult to score, and extra training may be required in order to make scores on these items meaningful. In analyses of training and mastery testing of FIM items, the problem solving item in particular led to difficulty in scoring immediately after training. ${ }^{16}$ Discussions with the FIM coordinators confirmed that considerable confusion exists about these items.

In the data across the four subgroups, a discipline-specific interpretation of discrepancies is possible: three of the four items in group 1 are collected by physical therapists; four of the six items in group 2 are collected by occupational therapists; with the exception of the stairs item, items in group 3 are collected by nurses (bladder and bowel management) and by speech therapists; and items in group 4 are collected by nurses at the ACRS and by social workers and psychologists at the ORS. As mentioned at the beginning of this study, these data were collected without benefit of a systematic, regularly scheduled training program across disciplines at the two institutions, although discipline-specific training was provided for the items each discipline was responsible for. Now that such a training program is in place, it will be important to repeat this analysis. Indeed, this study and its repetition can provide a useful pre- and post-test of the effectiveness of the new training program.

The dependent $t$-tests produced significant differences in scoring of patients more highly at the ACRS in most of the selfcare 
items and more highly in scoring at the ORS in the social cognition items. Again, patient motivation and rater error were confounded here, although it was apparently not a systematic effect for either, since some items were higher at one institution, and other items at the other site.

Finally, total FIM scores of various subgroups showed high reliability for burden of care scores for the complete and incomplete paraplegic and the complete quadriplegic groups of patients. However, the reliability coefficient was poor for the group of incomplete quadriplegic patients. Data collection continues for all patients, and with an increase in sample size, detailed analyses of these subgroups will be possible.

\section{Conclusions}

In summary, we offer the following suggestions for using individual FIM items for research with SCI patients:

1 An analysis of various kinds of ways of measuring agreement can be useful in studies of interinstitutional agreement of individual FIM items.

2 Reliability of the total FIM scores appears more than adequate for this sample of SCI patients, making the FIM an appropriately reliable instrument for measuring burden of care for SCI patients.

3 Research studies using the FIM items as outcome measures might give special consideration to the items in group 1 which show above average agreement using both reliability coefficients and proportion agreement measure.
4 We need to achieve a better understanding at the RSCICDV of the items which fall into group 2 (above average reliability coefficients, below average proportion agreement), specifically of the relative contributions made by patient motivation and rater motivation for these FIM items.

5 Items which show above average proportion agreement but below average reliability coefficients (group 3) do not present scoring difficulties, but the lack of variation in SCI patients may make them poor candidates for measuring outcomes in SCI patients.

6 Training should focus on items which show below average agreement and below average reliability coefficients (group 4 in this study).

7 It may be more difficult to rate some subgroups of SCI patients (in these data, incomplete quadriplegic patients) than others. More data need to be collected to begin to analyze individual FIM items within various populations of SCI patients to understand why this group seems different from the other three. We need to collect more data, do more analyses, and continue to refine our methodological tools in order to achieve a clearer understanding of the role of individual FIM items in interinstitutional research.

\section{Acknowledgement}

This study was supported in part by an award from the National Institute on Disability and Rehabilitation Research to the Regional Spinal Cord Injury Center of Delaware Valley, \#H133N00027.

\section{References}

1 Guide for Use of the Uniform Data Set for Medical Rehabilitation (1990) Center for Functional Assessment Research, State University of New York at Buffalo, Buffalo, NY.

2 Whiteneck G (1988) A Functional Independence Measure trial in spinal cord injury model systems. Abstracts Digest, American Spinal Injury Association 14th Annual Scientific Meeting 1988: 48.

3 Shepard MJ (NASCIS III Coordinator) (1992) Personal communication.

4 Segal M (1992) Analysis of the incidence of falls in the falls risk assessment program. Submitted to Thomas Jefferson University Utilization Review, Philadelphia PA.

5 Heinemann AW, Linacre M, Hamilton B, Wright B, Mamott B (1991) Rating scale analysis of the Functional Independence Measure. J Am Paraplegia Soc 14: 86.

6 Kaplan RM, Saccuzzo DP (1989) Psychological Testing: Principles, Applications, and Issues. 2nd ed. Brooks/Cole, Pacific Grove, PA.

7 Ditunno JF (1992) New spinal cord injury standards. Paraplegia 30: 90-91.

8 Ditunno JF (1992) Functional assessment measures in CNS trauma. J Neurotrauma 9: S301-S305. 
9 Dollfus P (1992) The scientific committee of the International Medical Society of Paraplegia. Paraplegia. 30: 22.

10 Hamilton B, Laughlin JA. Granger CV, Kayton RM (1991) Interrater agreement of the seven level Functional Independence Measure (FIM). Arch Phy's Med Rehabil 72: 790.

11 Anastasi A (1982) Psychological Testing. 5th ed. Macmillan, New York.

12 Spearman CE (1904) The proof and measurement of association between two things. Am J Psychol 15: $72-101$.

13 Cohen J (1960) A coefficient of agreement for nominal scales. Educ Psychol Measurement 20: 37-46.

14 Feinstein AR, Cicchetti DV (1990) High agreement but low kappa: I. The problems of two paradoxes. $J$ Clin Epidemiol 43: 543-549.

15 Davidoff GN, Roth EJ, Haughton JS, Ardner MS (1990) Cognitive dysfunction in spinal cord injury patients: Sensitivity of the Functional Independence Measure subscales vs neuropsychologic assessment. Arch Phys Med Rehabil 71: 326-329.

16 Segal M, DeJoseph L (1992) FIM mastery testing: videotapes vs. written case studies. Presentation at the American Paraplegia Society 38th annual conference, Las Vegas, 1992. 